\title{
On the $\mathbf{N}$ non-attacking queens problem
}

\author{
Aldous Cesar F. Bueno \\ Department of Mathematics and Physics, Central Luzon State University \\ Science City of Muñoz 3120, Nueva Ecija Philippines \\ E-mail address: aldouz_cezar@yahoo.com
}

\begin{abstract}
In this paper, a new version of the proof of the n-queens problem was presented. A new condition for the diagonal conflicts between the queens was added for the proof of the theorem.
\end{abstract}

Keywords: $N$-Queens problem, $N$ non-attacking queens on an $N x N$ board.

\section{Introduction}

The 8-queens problem is a classic chess problem that first appeared in the German newspaper Schachzeitung in September 1848. It was posed under a pseudonym by Max Bezzel who is a chess player. By 1854, 40 different solutions to this problem had been published on the same newspaper.

This problem was also posed in another German newspaper by Franz Nauck in June 1850. He correctly published the 92 possible solutions on the same newspaper without any proof that his list was complete. Carl Friedrich Gauss read Nauck's account of this problem in the summer of the same year and by September 1, wrote to a friend that he found 76 solutions. Many historical accounts credit the solutions to this problem to Gauss and hence producing a historical error that repeats over and over again.

The 8-queens problem asks to put 8 queens on the chessboard such that none of them attacks any of the others. It seems impossible because a queen on one of the 4 center squares of the chessboard can dominate 27 squares. Furthermore, a minimum of 5 queens can dominate the chessboard.

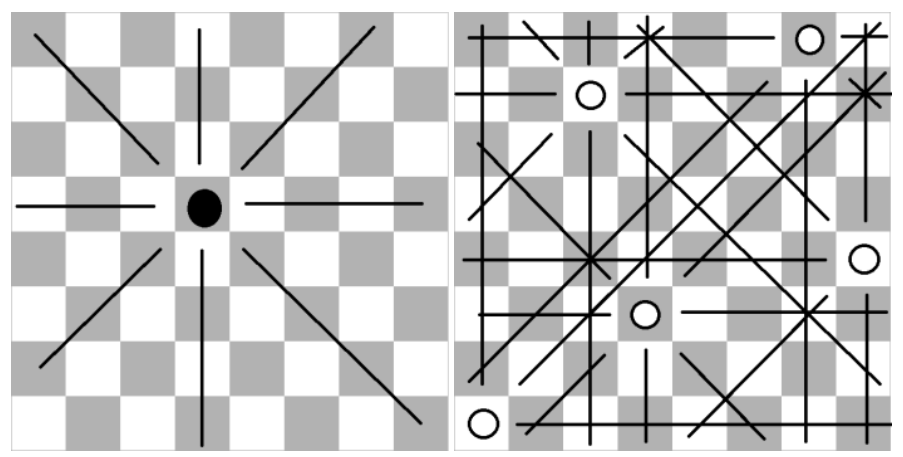

Fig. 1: A queen on one of the 4 center squares of chessboard and 5 queens dominating the whole board

On the 92 solutions, 12 are fundamental and the others are generated by their rotations and reflections. 

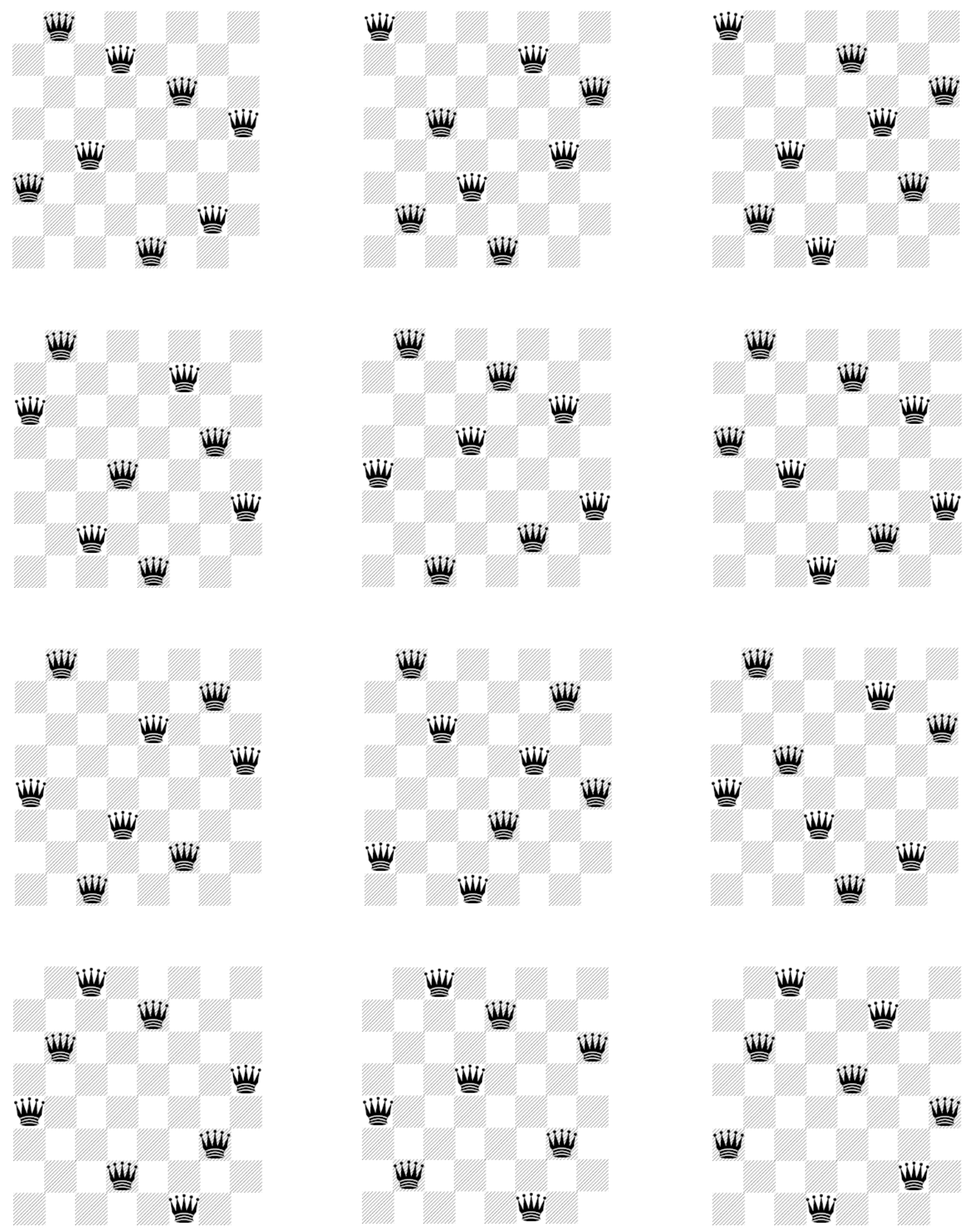

Fig. 2: The 12 fundamental solutions

\section{The N-Queens problem}

The $\mathrm{N}$-queens problem is the generalization of the 8-queens problem. Now, it asks to put $\mathrm{N}$ queens on an NxN board. The problem was proven to have solutions for all values of $\mathrm{N}$ except for $\mathrm{N}=2$ and $\mathrm{N}=3$. A queen alone can dominate the $2 \times 2$ board and also a $3 \times 3$ board. Two queens can also dominate the $3 \times 3$ board. 

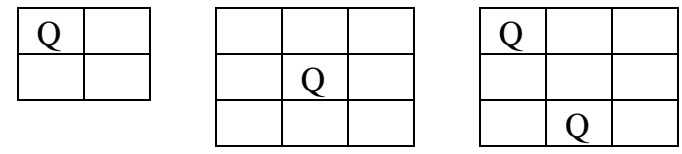

Fig 3: $2 \times 2$ board dominated by a queen and $3 \times 3$ boards dominated by a queen and 2 queens

In 1910, Wilhelm Ahrens had proven that there are solutions to the N-queens problem for all $\mathrm{N}$ not equal to 2 and 3. Many others also proved the existence of solutions to this problem.

\section{Main result}

Theorem 3.1: For $N>3$ there is at least a solution to the $N$-queens problem.

This proof is my own version. The second condition added is for the diagonal conflicts between the queens.

\section{Proof:}

First, write queens solution as a function $\mathrm{f}(\mathrm{k}), \mathrm{k}=0,1,2, \ldots, \mathrm{N}-1$, so that the $\mathrm{kth}$ queens is placed at the $(k, f(k))$ coordinate of the chessboard.

Need to be shown:

1) $\mathrm{f}$ is 1-1: for showing that no queens are in the same row and column.

2) $|\mathrm{f}(\mathrm{k})-\mathrm{f}(\mathrm{j})| \neq|\mathrm{k}-\mathrm{j}|$ for every $\mathrm{k} \neq \mathrm{j}$ where $\mathrm{k}, \mathrm{j}$ ranges from $0-\mathrm{N}-1$ : for showing that no queens are in the same diagonal.

Both should be satisfied by the function.

The proof splits up according to the residue class of $\mathrm{N}(\bmod 6)$

\section{CASE A: $N=6 m+1$ or $N=6 m+5$}

In this case, $\mathrm{f}(\mathrm{k})=2 \mathrm{k}(\bmod \mathrm{N})$

For $\mathrm{f}$ to be $1-1$, we need to show that $f(k)=f(j) \rightarrow k=j$

$f(k)=f(j)$

$2 \mathrm{k}(\bmod \mathrm{N})=2 \mathrm{j}(\bmod \mathrm{N})$

Note that:

$\mathrm{f}(\mathrm{k})=2 \mathrm{k}(\bmod \mathrm{N}) \rightarrow \mathrm{f}(\mathrm{k})-2 \mathrm{k}=0(\bmod \mathrm{N}) \rightarrow \mathrm{f}(\mathrm{k})-2 \mathrm{k}=\mathrm{Np}, p \in \mathbb{Z}$

Thus,

$2 \mathrm{k}+\mathrm{Np}=2 \mathrm{j}+\mathrm{Np}$

$2 \mathrm{k}=2 \mathrm{j}$

$\mathrm{k}=\mathrm{j}$

Therefore $\mathrm{f}$ is $1-1$ so no queens are in the rows and columns.

Next is to show that no queens are in the same diagonals, that is to show that $|f(k)-f(j)| \neq|k-j|$ where $\mathrm{k} \neq \mathrm{j}$.

Assume that $\mathrm{k} \neq \mathrm{j}$, now

$|\mathrm{f}(\mathrm{k})-\mathrm{f}(\mathrm{j})|=|\{2 \mathrm{k}(\bmod \mathrm{N})\}-\{2 \mathrm{j}(\bmod \mathrm{N})\}|$

Recall that:

$\mathrm{f}(\mathrm{k})=2 \mathrm{k}(\bmod \mathrm{N}) \rightarrow \mathrm{f}(\mathrm{k})-2 \mathrm{k}=0(\bmod \mathrm{N}) \rightarrow \mathrm{f}(\mathrm{k})-2 \mathrm{k}=\mathrm{Np}, p \in \mathbb{Z}$

Hence, the following will be obtained

$|2 \mathrm{k}+\mathrm{Np}-(2 \mathrm{j}+\mathrm{Nq})|=|2(\mathrm{k}-\mathrm{j})+\mathrm{N}(\mathrm{p}-\mathrm{q})| ; p, q \in \mathbb{Z}$

Suppose $|2(k-j)+N(p-q)|=|k-j|$

$\Rightarrow 2(\mathrm{k}-\mathrm{j})+\mathrm{N}(\mathrm{p}-\mathrm{q})=\left\{\begin{array}{l}k-j ; k-j \geq 0 \\ -(k-j) ; k-j<0\end{array}\right.$ 
For the first case:

$2(k-j)+N(p-q)=k-j \rightarrow k-j+N(p-q)=0 \rightarrow k-j=N(q-p) \rightarrow \frac{k-j}{N}=q-p$

This leads to a contradiction since $\mathrm{q}-\mathrm{p}$ is an integer and $\frac{k-j}{N}$ isn't.

For the second case:

$2(k-j)+N(p-q)=-(k-j) \rightarrow 3(k-j)+N(p-q)=0 \rightarrow 3(k-j)=N(q-p)$ $\rightarrow \frac{3(k-j)}{N}=q-p$

Again, a contradiction had arisen, since $\mathrm{q}-\mathrm{p}$ is an integer and $\frac{3(k-j)}{N}$ isn't. It's wrong to assume that $|f(k)-f(j)|=$ $|k-j|$. Therefore, $|f(k)-f(j)| \neq|k-j|$ and this means that there are no diagonal conflicts between the queens.

\section{CASE B: $\mathrm{N}=6 \mathrm{~m}$ or $\mathrm{N}=6 \mathrm{~m}+4$}

If this is the case, then one takes the solution of (Case A) for the $(\mathrm{N}+1) \mathrm{x}(\mathrm{N}+1)$ board and removes the queen in the $(0,0)$ position (i.e. the leftmost column and bottom row). The result is an $\mathrm{N} \mathrm{x} \mathrm{N}$ solution.
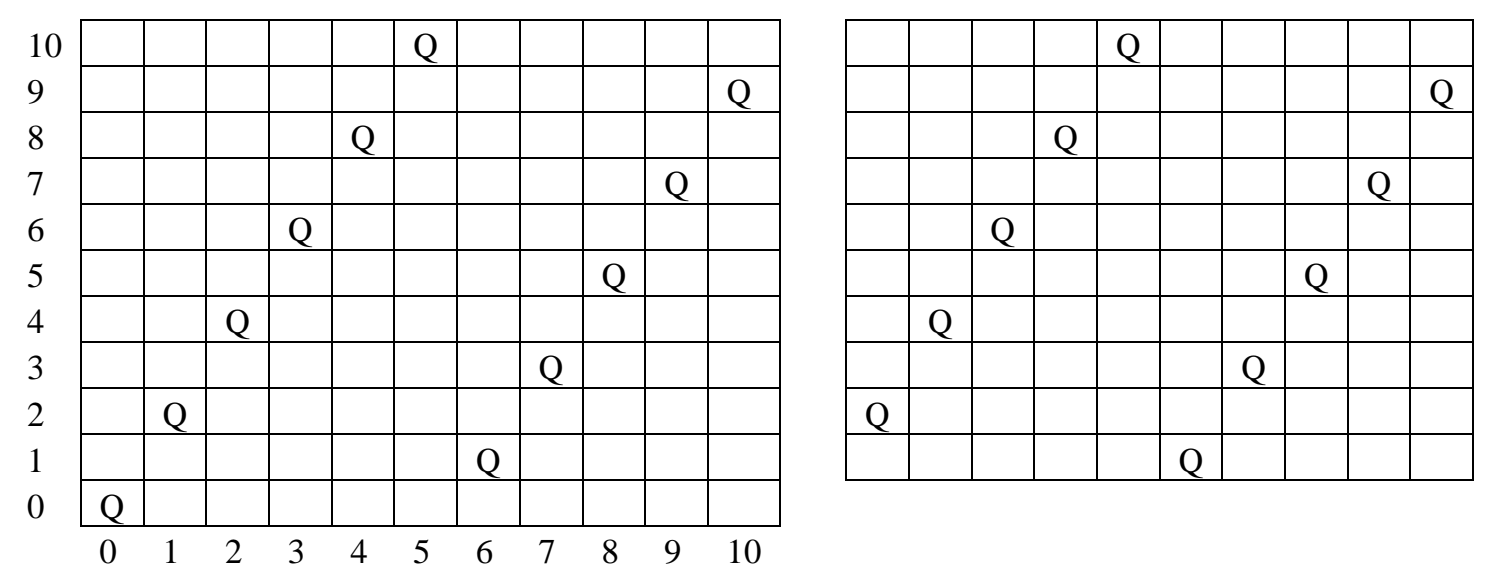

\section{CASE C: $\mathrm{N}=6 \mathrm{~m}+2$ or $\mathrm{N}=6 \mathrm{~m}+3$}

For $6 \mathrm{~m}+2$, put a queen at $(\mathrm{k}, \mathrm{f}(\mathrm{k}))$ where:

$f(k)=\left\{\begin{array}{l}2 k+\frac{N-2}{2}(\bmod N) ; 0 \leq k \leq \frac{N-2}{2} \\ N-1-f(N-1-k) ; \frac{N}{2} \leq k \leq N-1\end{array}\right.$

The following are the cases for testing if $\mathrm{f}$ is $1-1$ :

$k, j \in\left[0, \frac{N-2}{2}\right], k, j \in\left[\frac{N}{2}, N-1\right]$ and $k \in\left[0, \frac{N-2}{2}\right], j \in\left[\frac{N}{2}, N-1\right]$

First case: $k, j \in\left[0, \frac{N-2}{2}\right]$

$f(k)=f(j) \rightarrow 2 k+\frac{N-2}{2}(\bmod N)=2 j+\frac{N-2}{2}(\bmod N)$

$\rightarrow 2 k+\frac{N-2}{2}+N p=2 j+\frac{N-2}{2}+N p \rightarrow 2 k=2 j \rightarrow k=j$

Second case: $k, j \in\left[\frac{N}{2}, N-1\right]$

$f(k)=f(j) \rightarrow N-1-f(N-1-k)=N-1-f(N-1-j)$ but $0 \leq N-1-k \leq \frac{N-2}{2}$

$\rightarrow 2(N-1-k)+\frac{N-2}{2}(\bmod N)=2(N-1-j)+\frac{N-2}{2}(\bmod N)$

$\rightarrow 2 N-2-2 k+\frac{N-2}{2}+N p=2 N-2-2 j+\frac{N-2}{2}+N p$

$\rightarrow-2 k=-2 j \rightarrow k=j$ 
Last case: $k \in\left[0, \frac{N-2}{2}\right], j \in\left[\frac{N}{2}, N-1\right]$

$f(k)=f(j) \rightarrow 2 k+\frac{N-2}{2}(\bmod N)=N-1-f(N-1-j)$

$\rightarrow 2 k+\frac{N-2}{2}+N p=N-1-\left\{2(N-1-j)+\frac{N-2}{2}(\bmod N)\right\}$

$\rightarrow 2 k+\frac{N-2}{2}+N p=N-1-\left\{2(N-1-j)+\frac{N-2}{2}+N q\right\}$

$\rightarrow 2 k-2 j+2 N-3=N p-N q$

$\rightarrow k-j=\frac{3-N(p+q+2)}{2}$

$\rightarrow k-j=\frac{3-(6 m+2)(p+q+2)}{2}$

$\rightarrow k-j=\frac{3}{2}-(3 m+1)(p+q+2)$

This leads to a contradiction because the right side of the equality is not an integer while the left side is always an integer.

Thus $\mathrm{f}$ is $1-1$ and no queens are on the same row or column.

For the second condition, the same cases will also be considered.

First case: $k, j \in\left[0, \frac{N-2}{2}\right]$

$|f(k)-f(j)|=\left|\left\{2 k-\frac{N-2}{2}(\bmod N)\right\}-\left\{2 j-\frac{N-2}{2}(\bmod N)\right\}\right|$

$=\left|\left\{2 k-\frac{N-2}{2}+N p\right\}-\left\{2 j-\frac{N-2}{2}+N q\right\}\right|$

$=|2(k-j)+N(p-q)| \neq|k-j|$

By Case A, this is a contradiction.

Second case: $k, j \in\left[\frac{N}{2}, N-1\right]$

$|f(k)-f(j)|=|\{N-1-f(N-1-k)\}-\{N-1-f(N-1-j)\}|$

$=|f(N-1-j)-f(N-1-k)|$

$=\left|\left\{2(N-1-j)-\frac{N-2}{2}(\bmod N)\right\}-\left\{2(N-1-k)-\frac{N-2}{2}(\bmod N)\right\}\right|$

$=\left|\left\{2(N-1-j)-\frac{N-2}{2}+N q\right\}-\left\{2(N-1-k)-\frac{N-2}{2}+N p\right\}\right|$

$=|2(k-j)+N(q-p)| \neq|k-j|$

which has the same result as that of the previous case that leads to contradiction.

The last case may seem difficult but it also leads to contradiction.

Last case: $k \in\left[0, \frac{N-2}{2}\right], j \in\left[\frac{N}{2}, N-1\right]$

$|f(k)-f(j)|=\left|\left\{2 k-\frac{N-2}{2}(\bmod N)\right\}-\{N-1-f(N-1-j)\}\right|$

$=\left|\left\{2 k-\frac{N-2}{2}+N p\right\}-\left\{N-1-2(N-1-j)+\frac{N-2}{2}(\bmod N)\right\}\right|$

$=\left|\left\{2 k-\frac{N-2}{2}+N p\right\}-\left\{N-1-2(N-1-j)+\frac{N-2}{2}+N q\right\}\right|$

$=|2(k-j)+N(p+q+2)-3|$

Suppose $|2(k-j)+N(p+q+2)-3|=|k-j|$

$\rightarrow 2(k-j)+N(p+q+2)-3=\left\{\begin{array}{c}k-j ; k \geq j \\ -(k-j) ; k<j\end{array}\right.$

$* 2(k-j)+N(p+q+2)-3=k-j \rightarrow k-j+3=-N(p+q+2)$ 
$* 2(k-j)+N(p+q+2)-3=-(k-j) \rightarrow k-j+1=-\frac{N(p+q+2)}{3}$

In the first equality, if we divide by $\mathrm{N}$, the left side will be $(\mathrm{k}-\mathrm{j}+3) / \mathrm{N}$ which is not always an integer while the right side which is $-(\mathrm{p}+\mathrm{q}+2)$ which is always an integer, thus a contradiction.

In the last equality, if we multiply by $3 / \mathrm{N}$, the left side becomes $3(\mathrm{k}-\mathrm{j}+1) / \mathrm{N}$ which is not always an integer while the right side is $-(\mathrm{p}+\mathrm{q}+2)$ which is always an integer, a contradiction.

So it's wrong to assume that $|\mathrm{f}(\mathrm{k})-\mathrm{f}(\mathrm{j})|=|\mathrm{k}-\mathrm{j}|$. Therefore, there are no queens on the same diagonals. Furthermore, this solution does not have a queen on the main diagonal. One can construct a $6 m+3$ solution by adding a row and a column to the edge of the board and putting a queen on the new corner.

This completes the proof.

\section{Conclusion}

In this paper, we have shown that the N-queens problem can also be proven by adding the condition $|f(k)-f(j)| \neq|k-j|$ for every $\mathrm{k} \neq \mathrm{j}$ where $\mathrm{k}, \mathrm{j}$ ranges from $0-\mathrm{N}-1$. Also, from the proof of Theorem 3.1, other solutions for the $\mathrm{N}$-queens problem can be formed from other solutions with different dimensions.

\section{References}

[1] H. A. Noon, "Surreal Numbers and the N-Queens Game", Bachelors Thesis, Bennington College, Bennington, Vermont.

[2] H. A. Noon, G. V. Brummelen, "The Non-attacking Queens Game", The College Mathematics Journal, Vol. 37 No. 3, May 2006.

[3] Z. Szaniszlo, M. Tomomva and C. Wiyels, "The N-Queens Problem on a symmetric Toeplitz matrix", Elsevier, Discrete Mathematics 309(2009) 969-974. 